\title{
The Effectiveness of a Care Transitions and Rapid Response Nurse Intervention at Reducing Readmissions and Emergency Department Use for High Risk Patients
}

\author{
David Raymong McNeil ${ }^{1 *}$, Roger Strasser ${ }^{2}$, Nancy Lightfoot ${ }^{3}$ and Raymond Pong ${ }^{4}$ \\ ${ }^{1}$ Department of Clinical Transformation and chief nursing executive at Health Sciences North in Sudbury, ON, Canada \\ ${ }^{2}$ Northern Ontario School of Medicine, at Laurentian University and Lakehead University, in Thunder Bay, ON, Canada \\ ${ }^{3}$ School of Rural and Northern Health, at Laurentian University, in Sudbury, ON, Canada \\ ${ }^{4}$ Centre for Rural and North Health Research, at Laurentian University, in Sudbury, ON, Canada
}

"Corresponding author: David Raymong McNeil, Vice President Patient Services and CNE, Health Sciences North, Nursing Administration, 41 Ramsey Lake RD, Sudbury, ON P3E 5J1, Canada, Tel: 705-561-6426; E-mail: dmcneil@hsnsudbury.ca

Received date: August 21, 2017; Accepted date: October 12, 2017; Published date: October 20, 2017

Copyright: @ $2017 \mathrm{McNeil}$ DR, et al. This is an open-access article distributed under the terms of the Creative Commons Attribution License, which permits unrestricted use, distribution, and reproduction in any medium, provided the original author and source are credited

\begin{abstract}
The transition from hospital to home is a vulnerable period for the elderly patient with complex conditions, who are often frail, at risk for adverse events and unable to navigate a system of poorly coordinated care in the postdischarge period.

This article presents the results of a randomized control trial evaluating the effectiveness of an intervention involving a care transitions nurse and a rapid response nurse at lengthening the time to first readmission, emergency department use and total hospital bed days during the 30 days, 60 days and 90 days post-discharge periods for patients at high risk of readmission. The intervening impact of social isolation and patient frailty was also evaluated.

No statistically significant differences were found between the intervention and control groups on the time to first readmission or in the post-discharge emergency department or inpatient bed use. Social isolation and frailty were not shown to significantly influence these outcomes.
\end{abstract}

Keywords: Care transitions; Rapid response; Frailty; Integration; Readmissions

\section{Care transitions, Rapid Response, Frailty, Integration, Readmissions}

Patients are being discharged more quickly, allowing less time for providers to prepare patients and their families for discharge [1-4]. Patients are more medically complex [3] and are discharged before they have completely recovered [5-7].

Achieving seamless transitions and coordination of post-discharge care is challenging given the high degree of system fragmentation [8]. Medical specialization and organizational separation between community, hospitals and physician services creates a degree of system complexity that increases the probability of failed communication between providers and discontinuity in care $[3,9]$. The information transition from one provider to another is often incomplete as the patient moves from one care setting to another $[3,6,10]$.

While achieving seamless transitions between care settings is viewed as crucial to high-quality care particularly for the frail older person; adverse events, at the point of transition, occur with regular frequency [11-14]. These include: medication errors, communication breakdowns between providers, incomplete and inaccurate transfer of information, inadequate patient and caregiver education, complex and poorly understood discharge instructions, errors in follow-up of diagnostic tests, infections, falls and complications related to procedures and limited access to services [12,15-17].

Hospitals traditionally have viewed discharge as a transaction, signifying an end point, where they are done with the patient, letting others, whether it be the family or other community providers, deal with often unresolved or unplanned for issues [18-20]. In recent years, significant interest has developed around the implementation of care transition interventions, as care coordinating mechanisms, to reduce avoidable readmissions and other adverse events in the post-discharge period [3,21-28].

\section{Study Purpose and Overview}

This study was completed in two phases. The first phase involved a randomized control trial which tested the efficacy of an intervention involving a care transitions nurse and a rapid response nurse plus home care follow and referral to a chronic disease management clinic. The aim was to lengthen the time to first readmission or emergency department visit and reduce total post-discharge inpatient and emergency department use within the time intervals of 30 days, 60 days and 90 days from the time of discharge of the index admission. It is the results of the randomized trial that are presented in this study. The second phase involved a qualitative analysis using focus groups and individual interviews, involving providers, to identify the factors that facilitated or were barriers to the implementation of this care 
transitions intervention. These results are presented elsewhere (Author's Article).

\section{Methods}

The research was approved by the Research Ethics Boards of Laurentian University, Health Sciences North and the Northeast Community Care Access Centre.

Eligible patients in the randomized trial were selected from a population of patients admitted from home or a home-like setting between June 2013 and June 2014 to an academic health sciences centre located in the City of Greater Sudbury in north-eastern Ontario, Canada. Patients were admitted from the emergency department and had at least one admission in the last 12 months with one or more of the following chronic conditions: congestive heart failure (CHF), chronic obstructive pulmonary disease (COPD), diabetes or dementia. The LACE index was completed on all patients to identify the patient's risk for readmission. The LACE index uses the criteria of: length of stay (L); acuity (A); comorbidity (C); and emergency department use (E) [29]. Patients with a LACE index of $>10$ were eligible for enrolment. Excluded were patients admitted from a long-term care, chronic care facility or patients with severe cognitive impairment or a mental health or palliative diagnosis and patients unable to consent.

Patients meeting the inclusion criterion were randomized to either the intervention group or the control group. A stratified blocked randomization process was used for assignment [30]. Patients, care providers and the researcher were unaware of the patient's assignment prior to enrolment.

Patients in the control group received usual discharge planning and home follow-up support. For patients in the intervention group, a patient-centred care plan and discharge plan was developed and comprehensive in-hospital teaching completed using teach-back as well as a medication reconciliation completed by the hospital's care transitions nurses.

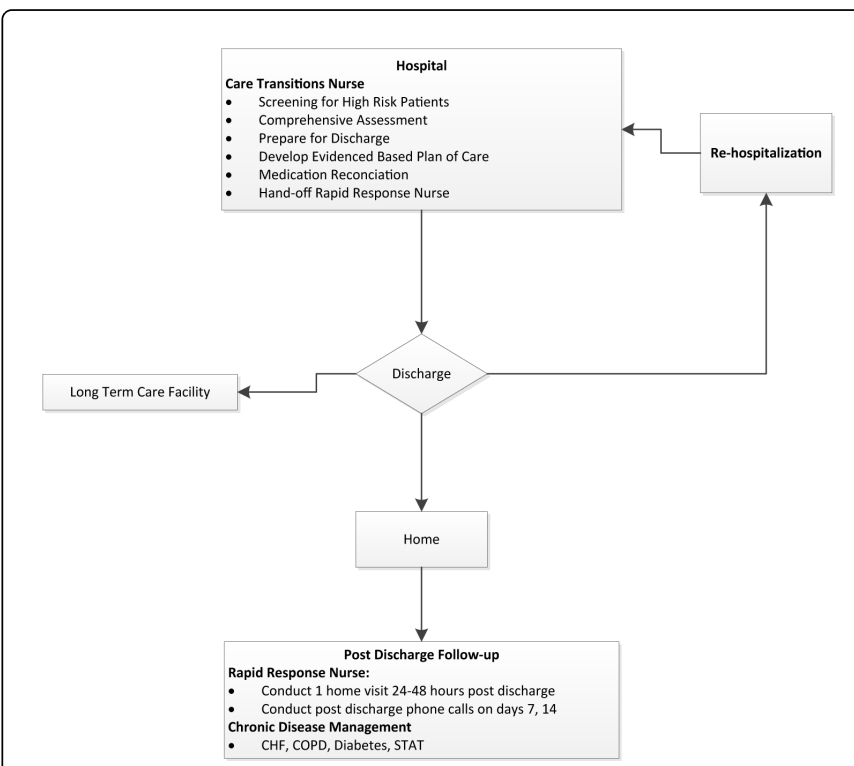

Figure 1: Overview of care transition and rapid response intervention.
A hand-off occurred between the hospital-based care transitions nurse and a community-based rapid response nurse. The rapid response nurse conducted an in-home follow-up visit within 24 to $48 \mathrm{~h}$ after discharge. Additional in-home and telephone follow-up was provided by the rapid response nurse for a period of up to 30 days post-discharge. Patients randomized to the intervention group were referred for follow-up at one or more of the following chronic disease management clinics for diabetes, CHF or COPD. High-risk patients without access to a primary care provider were referred to the Shortterm Assessment and Treatment (STAT) service at the hospital (Figure $1)$.

\section{Data Collection Methods and Instruments}

The frailty index of all patients was measured using the Comprehensive Geriatric Assessment, Frailty Index web-based version (Videx: FI-CGA) [31] their social isolation score was measured using the Friendship Scale [32] and their satisfaction with the transitions was measured using the15-item Care Transitions Measure administered via telephone at least 30-days post-discharge by a research assistant blinded to the patient's assignment [33]. Other data collected included the number of inpatient admissions and emergency department visits in the 6-month period prior to the index admission, the number and length of stay of hospital admissions and emergency department visits in the post-discharge period, admission date, visit dates and discharge dates of intervention group patients who received the communitybased rapid response nursing service.

\section{Analysis}

The data analysis was completed based on the intention-to-treat principle using IBM SPSS Version 21 (2012) software [34]. The time to first readmission and the time to first emergency department visit were analyzed using the Kaplan-Meier Survival Analysis. The Fisher exact test was used to compare the differences in utilization rates, between the intervention and control groups for the number of readmissions, emergency department visits at 30 days, 60 days and 90 days into the post-discharge period. The Mann-Whitney $\mathrm{U}$ test was used to compare total bed-days for the same periods. Two-way analysis of variance was used to determine if there was intervening impact of frailty and social isolation on post-discharge utilization (Table 1).

\begin{tabular}{|l|l|l|}
\hline Randomized Assignment & Intervention $\mathbf{n}(\%)$ & Control $\mathbf{n}(\%)$ \\
\hline Characteristic & 136 & 133 \\
\hline $\begin{array}{l}\text { Age (Mean, M, Standard \& Deviation, } \\
\text { SD) }\end{array}$ & $75.11+/-10.9$ & $75.21+/-10.4$ \\
\hline Gender & & \\
\hline Male & $65(47.8)$ & $58(43.6)$ \\
\hline Female & $71(52.2)$ & $75(56.3)$ \\
\hline Lives Alone & & \\
\hline Yes & $51(37.5)$ & $54(40.6)$ \\
\hline No & $85(62.5)$ & $79(59.4)$ \\
\hline Living Location & & \\
\hline Apartment & $42(30.9)$ & $45(33.8)$ \\
\hline House & $65(47.8)$ & $64(48.1)$ \\
\hline
\end{tabular}


Citation: McNeil DR, Strasser R, Lightfoot N, Pong R (2017) The Effectiveness of a Care Transitions and Rapid Response Nurse Intervention at Reducing Readmissions and Emergency Department Use for High Risk Patients. J Nurs Care 6: 429. doi:10.4172/2167-1168.1000429

Page 3 of 7

\begin{tabular}{|c|c|c|}
\hline Assisted Living & $9(6.6)$ & $11(8.3)$ \\
\hline Retirement Home & $12(8.8)$ & $7(5.3)$ \\
\hline Extended Family & $8(5.8)$ & $6(4.5)$ \\
\hline \multicolumn{3}{|l|}{ Marital Status } \\
\hline Married & $58(38.2)$ & 57 (42.9) \\
\hline Common Law & $2(1.5)$ & $1(0.8)$ \\
\hline Divorced & $11(7.3)$ & $11(8.3)$ \\
\hline Single & $16(11.8)$ & $14(10.5)$ \\
\hline Widow & $49(36.0)$ & $50(37.6)$ \\
\hline \multicolumn{3}{|l|}{ Number of Medications } \\
\hline$<6$ & $2(1.5)$ & $10(7.5)$ \\
\hline$>5<10$ & $23(16.9)$ & $6(4.5)$ \\
\hline$>10<20$ & $89(65.4)$ & $59(44.4)$ \\
\hline$>20$ & $22(16.2)$ & $58(43.6)$ \\
\hline \multicolumn{3}{|l|}{ Primary Care Physician } \\
\hline Yes & $121(89.0)$ & $118(88.7)$ \\
\hline No & $15(11.0)$ & $15(11.3)$ \\
\hline Number of Chronic Diseases & $\begin{array}{l}(\mathrm{M}=4.48 \\
\mathrm{SD}=1.755)\end{array}$ & $\begin{array}{l}(\mathrm{M}=4.34 \\
\mathrm{SD}=1.723)\end{array}$ \\
\hline 1 & $14(10.3)$ & $10(7.5)$ \\
\hline 2 & $12(8.8)$ & $16(12.0)$ \\
\hline 3 & $10(7.4)$ & $20(15.0)$ \\
\hline 4 & $20(14.7)$ & $13(9.8)$ \\
\hline 5 & $19(14.0)$ & $21(15.8)$ \\
\hline$>5$ & $61(44.85)$ & $53(39.9)$ \\
\hline \multicolumn{3}{|l|}{ Congestive Heart Failure } \\
\hline Yes & $64(47.1)$ & $67(50.4)$ \\
\hline No & $72(52.9)$ & 66 (49.6) \\
\hline \multicolumn{3}{|l|}{ Diabetes } \\
\hline Yes & $66(48.5)$ & $67(50.4)$ \\
\hline No & $70(51.5)$ & $66(49.6)$ \\
\hline \multicolumn{3}{|l|}{ COPD } \\
\hline Yes & $82(60.3)$ & $75(56.4)$ \\
\hline No & $54(39.7)$ & $58(43.6)$ \\
\hline \multicolumn{3}{|l|}{ Dementia } \\
\hline Yes & $31(22.8)$ & $23(17.3)$ \\
\hline No & $108(77.2)$ & $106(82.7)$ \\
\hline LACE & $\begin{array}{l}M=13.750 \\
S D=2.090\end{array}$ & $\begin{array}{l}M=13.870 \\
S D=2.302\end{array}$ \\
\hline
\end{tabular}

\begin{tabular}{|l|l|l|}
\hline Friendship Score (Social Isolation) & $\begin{array}{l}M=17.60, \\
S D=6.225\end{array}$ & $\begin{array}{l}M=18.18, \\
S D=6.014\end{array}$ \\
\hline \multirow{2}{*}{ Frailty } & $M=0.591$, & $M=0.592$, \\
& $S D=0.124$ & $S D=0.119$ \\
\hline
\end{tabular}

Table 1: Demographic and health characteristics, intervention and control groups.

\section{Previous Health System Utilization}

The patient population enrolled in the trial experienced frequent inpatient admission and emergency department use in the six months preceding entry into the trial, with $32 \%$ of the intervention and $25 \%$ of the control group having more than one admission and $23 \%$ of the intervention and $26 \%$ of the control having more than one emergency department visit in the previous six months. This information is summarized in Table 2 below.

\begin{tabular}{|l|l|l|}
\hline Characteristic & $\begin{array}{l}\text { Intervention } \\
(\%) n=136\end{array}$ & $\begin{array}{l}\text { Control } \mathbf{n}(\%) \\
\mathbf{n}=133\end{array}$ \\
\hline $\begin{array}{l}\text { Previous Readmissions } \\
\text { last } \mathbf{6} \text { months }\end{array}$ & $(\mathrm{M}=1.500, \mathrm{SD}=0.8861)$ & $(\mathrm{M}=1.059, \mathrm{SD}=1.434)$ \\
\hline 1 & $92(67.7)$ & $98(73.7)$ \\
\hline 2 & $29(21.3)$ & $28(21.8)$ \\
\hline 3 & $8(5.9)$ & $3(2.3)$ \\
\hline 4 & $6(4.4)$ & $1(0.8)$ \\
\hline 5 & $0(0)$ & $1(0.8)$ \\
\hline 6 & $1(0.7)$ & $1(0.8)$ \\
\hline Previous ED Visits Last 6 & $(\mathrm{M}=4.48, \mathrm{SD}=1.755)$ & $(\mathrm{M}=1.093, \mathrm{SD}=1.535)$ \\
\hline Months & $59(43.4)$ & $63(47.4)$ \\
\hline 0 & $46(33.8)$ & $35(27.1)$ \\
\hline 1 & $14(10.3)$ & $12(9.02)$ \\
\hline 2 & $8(5.9)$ & $11(8.3)$ \\
\hline 3 & $4(2.9)$ & $5(3.8)$ \\
\hline 4 & $3(2.2)$ & $4(3.0)$ \\
\hline 5 & $2(1.5)$ & $2(1.5)$ \\
\hline$>5$ & & \\
\hline & & \\
\hline
\end{tabular}

Table 2: Previous 6 month admissions and emergency department visits.

The mean LACE index scores for the patients enrolled in the trial was 13.8 and predictive of a $19.8 \%$ to $23 \%$ probability of readmission or death within 30 days of discharge [29]. With a Friendship score mean of 17.89, the enrolled patients would best be characterized as marginally socially connected [32]. With a mean frailty score on admission of 0.59 , the study population could be considered as very frail [35]. 


\section{Post-discharge utilization}

In total, 251 of the 269 patients enrolled in the study were discharged home after their index admission. These 251 patients experienced a total of 272 readmissions during the study period. These readmissions resulted in the utilization of 5,197 bed days, representing the equivalent utilization of 11.7 inpatient hospital beds.

The mortality rate for those patients who entered the trial was $22.7 \%$ within a 12 months period. The mean frailty score for those who died was $(M=0.638, S D=0.104)$ and significantly higher than those who did $\operatorname{not}(\mathrm{M}=0.579, \mathrm{SD}=0.123), \mathrm{t}(267)=3.401, \mathrm{p}=0.001$.

The post-discharge inpatient readmission rates for all patients calculated from the discharge date of the index admission were: at 30 days, $22.3 \%$; at 60 days, $37 \%$; and at 90 days, $49 \%$. The post-discharge emergency department utilization rates for the same period were: at 30 days, $22.31 \%$; 60 days, $37.05 \%$ and 90 days, $49 \%$.

Based on the Fisher's exact test, no statistically significant differences were found between the intervention and control groups on the number of inpatient readmissions at 30 days, $(\mathrm{p}=0.76), 60$ days $(\mathrm{p}=0.79)$ and 90 days $(\mathrm{p}=0.90)$ or the number of emergency department visits at 30 days $(\mathrm{p}=0.63), 60$ days $(\mathrm{p}=0.68)$ or 90 days $(\mathrm{p}=0.54)$. No significant differences were found in the total hospital bed days used between the intervention and control groups based on the Mann Whitney $U$ at 30 days Median Intervention (6), Median Control (7), $U=370.5, p=0.390 ; 60$ days Median Intervention (6.5), Median Control (7), $\mathrm{U}=728, \mathrm{p}=0.791$ and 90 days Median Intervention (7), Median Control (8), $\mathrm{U}=1046.5, \mathrm{p}=0.783$.

\section{Time to first readmission and emergency department visit}

The time to first readmission and emergency department visit from the discharge date of the index admission was analysed using a Kaplan-Meier survival analysis. The median time to first readmission after the index discharge was 58 days. The median time to first emergency department visit was 52 days. Based on the log rank (Mantel-Cox) test, there was no statistically significant differences in the time to first readmission between the intervention and control groups (Figure 2; X2 $(1, \mathrm{~N}=251)=0.165, \mathrm{p}=0.685)$; nor were there any statistically significant differences in the time to first emergency department visit (Figure 3; X2 $(1, \mathrm{n}=251)=0.168, \mathrm{p}=0.682)$.

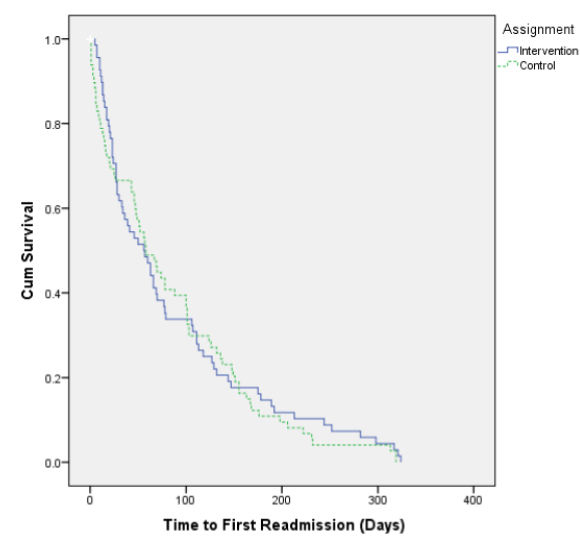

Figure 2: Time to first readmission, intervention vs. control.

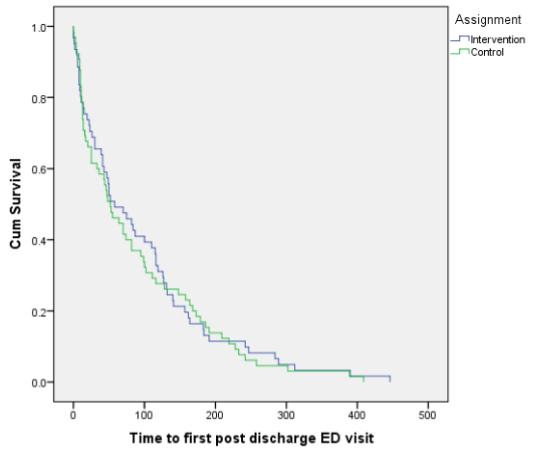

Figure 3: Time to first post discharge emergency visit (days).

\section{Impacts of frailty and social isolation}

No correlation was found between frailty and the time to first readmission, $\mathrm{r}(141)=-0.054, \mathrm{p}=0.531$, or the time to first emergency department visit, $\mathrm{F}(2,120)=0.691, \mathrm{p}=0.503$.

No differences were observed in the time to first readmission by friendship category, $\mathrm{F}(4,133)=2.366, \mathrm{p}=0.056$ or the time to first emergency department visit by friendship category, $\mathrm{F}(4,116)=1.754$, $\mathrm{p}=0.143$

Two-way analysis of variance demonstrated a main effect of gender, F $(1,119)=4.827, \mathrm{p}=0.03$, with females having a statistically significantly shorter time to first emergency department visit. There was no significant interaction effect of gender and living alone. F (1, $119)=0.022, p=0.882$. No statistically significant differences in time to first emergency department visit were found between those who lived alone and not alone, $\mathrm{F}(1,119)=0.018, \mathrm{p}=0.892$.

\section{Discussion}

This care transition intervention differed from others, such as Naylor's Care Transitions Model, Coleman's Care Transition Program $[3,24]$ and others which used a liaison or navigator roles to serve a system-spanning function, linking and coordinating care by facilitating the flow of information between care settings [36]. This particular study attempted to replicate the care coordination benefits of these previous studies by dedicating individuals within each organization the accountability for coordinating the development of the transitional care plan, the hand-off and post-discharge follow-up. The results of this particular study demonstrated that the intervention had no effect. The reason the intervention had no effect may be attributed to factors related to the patients, the design of the intervention/work process and/or the relationships between staff participating in the study. These are explored below.

\section{Patient Factors}

Patients selected for the intervention may have been simply too frail to have benefited from the intervention - a view supported by the providers who articulated this notion in the qualitative study reported elsewhere (Author's Article). Additionally, support for this assertion comes from comparing the results of this study with the work of Evans et al. [37]. 
The frailty index used in this trial is based on the counting of accumulated deficits in health, based on clinical signs, symptoms, diseases, laboratory and radiographic abnormalities. This information is typically collected on the basis of a comprehensive geriatric assessment $[35,38]$. The number of deficits a person has is expressed as a ratio of the total number of deficits measured to yield an individual score between 0 (no deficits) to 1 (all deficits present). The larger the number of deficits an individual possesses, the greater their potential for adverse health outcomes, including death. The index is calculated based on the presence or absence of deficits. An individual with 20 deficits would have a frailty score of $20 / 92=0.22$ [39]. Where an individual has accumulated two-thirds of the potential deficits, they are considered frail.

Evans et al. [37] tested the prognostic capacity of the frailty index in an acute care setting in relation to the risk of death, length of stay and discharge destination. Patients discharged home had the lowest admitting mean frailty index at 0.38 , compared to those who died (Frailty Index $=0.51$ ) and those discharged to a nursing home (Frailty Index $=0.49$ ). The mean frailty score for the population cohort in this trial was 0.59 , indicative of a highly frail population. The frailty of the population is corroborated by the finding that 18 of the patients enrolled in the trial were lost to death prior to discharge home and the overall mortality rate for the trial period was 22 . 7\%. A further indication of the frailty of the population was the mean LACE score for the population which was 13.9 , predictive of a $23 \%$ percent probability of mortality or hospital readmission within 30 days and consistent with the current trial's 30 -day readmission rate of $22.31 \%$ in the current trial [29]. Finally, the distribution of frailty scores in the trial followed a normal distribution. In work done by Mitnitski et al. [35] they found that a normal distribution of frailty scores in a population is consistent with one whose adaptive potential is considerably compromised, that is, systems having large number of "independent and uncompensated failures of subsystem and elements". According to Mitnitski et al. the distribution of well groups follows the gamma distribution [35].

While few trials have focused specifically on highly frail patients, in a mixed method study using a pre-post design, Ornstein et al. [40] applied a care transition intervention to a patient population that included patients with cognitive impairment, that were generally older with an average age of 81 years, more frail, requiring assistance with five or more activities of daily living and utilizing more than 8.2 medications. The intervention did not demonstrate a reduction in hospital readmissions or costs [40]. There is not yet consensus in the literature as to what patient population is most appropriate for what intervention. As pointed out by Hansen et al. [41], the significant challenge in evaluating care transition interventions are that populations have not been well defined in terms of objective measures, making comparability difficult [41].

The distribution of social isolation scores were different for men compared to women. Social isolation scores for men living alone were significantly lower (more isolated) than for men living with a significant other or extended family. On the main question of the intervening impact of social isolation on the time to first readmission, no differences were found by social isolation category nor were differences found between social isolation category and the time to first emergency department visit or total inpatient bed utilization by social isolation category. Friendship scores, that is, the measure of social isolation used in this study were not a predictor of health care utilization.
The time to first readmission analysis showed that women who lived alone had a statistically significantly shorter time-span to first readmission compared to men who lived alone. It may be that women are more likely to seek help earlier than men or that they have better social networks to encourage them to seek out care. The assertion that women living alone have a more fully developed social networks compared to men and have better social connectedness is supported in the literature $[42,43]$.

\section{Intervention Design}

A fundamental issue in the design of the intervention was that the home follow-up period was limited to 30 days. Given the frailty of the population, this was probably not sufficient. Many transitional interventions have longer periods of follow-up and have demonstrated longer-term success in changing health status and health utilization behaviours of the population they serve [44,45]. Naylor et al. [46] suggests that although transition interventions are successful in improving the patient's self-management capacity, there is a need for on-going follow-up in the post-discharge period given the on-going progressive nature of the chronic conditions of patients and in the case of this particular study, increasing levels of frailty.

This study differed from previous studies in that it implemented a hospital-to-home care transition initiative that spanned two different organizations. Involving two organizations is a function of the fragmented nature of Ontario's healthcare system where hospitals and home care agencies are separate entities. The initial study design contemplated utilizing a hospital-based care transition nurse in a liaison or navigator role, serving a system-spanning function, similar to work done elsewhere $[24,47]$. In the pilot phase of the trial, the care transitions nurse prepared the patient for discharge and followed the patient into the community for a period of up to 90 days. The pilot was found to be financially unsustainable for the hospital as it did not have sufficient resources to pay for all of the nursing hours required to implement the proposed model. As discussed in the literature, financial resource limitations represent a significant barrier to the implementation of care transition interventions [24,48].

The introduction of the Rapid Response Nurse Program by the Northeast Community Care Access Centre provided an opportunity to align the work of the care transition nurses with the rapid response nurses. To leverage available financial resources, the intervention was redesigned. The redesign of the work processes related to the information transfer protocols was left in the hands of the care transition and rapid response nursing teams and was led by the Coordinator of Care Transitions from the hospital and the Manager of Rapid Response from the Community Care Access Centre.

\section{Work Process Design}

A qualitative review was undertaken as part of this study. The detailed results of this review are presented elsewhere (Author's Article). These results provide insight into the challenges associated with the design of the work processes and relationships between professionals working in the two organizations and responsible for transitioning patients.

The data for the Qualitative study was collected through individual interviews and focus groups and the results analyzed using thematic content analysis. Based on the data collected it was found that the effectiveness of transitional coordination efforts was thwarted by ineffective communication which affected the quality of the underlying 
relationships between the two teams. Other barriers included: issues of role clarity, role awareness and lack acceptance of the positions within the respective organizations, the adequacy and reinforcement of coordinating mechanisms by the teams and management and the effectiveness of the information exchange protocols which were characterized as complex and cumbersome.

Another issue with the design of the intervention is that process and outcome measures were not shared with the teams on an on-going basis. This was not part of either the research design or program implementation strategy. The monitoring and tracking of shared process and outcome measures is one way in which alignment between the two teams might have been better achieved. The idea of creating alignment through measurement accountability has been suggested by others as a way to force the forging of partnerships between providers to create a greater degree of mutual accountability to each other, the patient and the system [49]. The assertion is that what gets measured drives leadership and organizational behaviour. Naylor et al. [46] argues for the expansion of publicly reported measures for both transitional care processes and outcomes as a way to generate real system change with measures which address patient and care-giver experience, with potentially avoidable readmissions and financial benefit identified as priority areas of measurement [46,48]. A more formally structured accountability relationship at the organizational (macro) level to better link the outcomes of the teams at the micro level to organizational outcomes and accountabilities may have been of benefit in the case of this intervention.

These challenges faced by both of the teams is reflected in the literature related to transitions and discharge [50,51]. Finally, as articulated in the work of others, the success of any care transitions intervention is the function of leadership [46]. During the course of this study there was leadership turnover and this clearly made the implementation more of a challenge.

\section{Implications}

This study raises several questions requiring further consideration in the design of transitional interventions particularly where the transition occurs between two different organizations. Careful consideration needs to be given to the intervention design, information transfer processes, organizational alignment and relationships and mutual organizational accountabilities. Achieving this requires strong leadership direction and on-going monitoring of performance. Additionally, consideration needs to be given to targeting the right intervention to the right patient population. Finally, there needs to be recognition that there will be an investment effect, that is, achieving the intended results of the intervention will take time.

\section{Limitations}

While there are several limitations, three major ones will be mentioned. Firstly, the results of the trial may not be generalizable to different population groups. The patient population in this study was generally very frail and the results might be different with a less frail population. Secondly, maintaining adherence to study protocols proved to be difficult. A total of 10 patients in the intervention group did not receive the intervention and 18 patients in the control group did receive the intervention. There is no evidence that this contamination was systematic. Finally, timeliness of primary care follow-up in the post-discharge period was not formally evaluated as part of this trial. Several studies have demonstrated that timely primary care follow-up in the post-discharge period is associated with the reduced risk of readmission [52,53]. This may have had an intervening impact on patient outcomes and would be a further area of research.

\section{Conclusion}

Effective patient transitions between organizations are achievable but are complex undertakings influenced by factors related to the patient, the design of the coordinating mechanism and relationship between professionals responsible for delivering care.

This randomized trail tested a care transitions and rapid response nurse intervention. Given the patient frailty and multi-morbidity the goals for the intervention may have been unrealistically high for a very old, frail, and sick patient cohort. The intervention may have been more effective with another patient population. An approach with the current patient population which focused on managing the palliative nature of their frailty may have been of more benefit. Modifications to the design of the coordinating mechanisms of the intervention are warranted with particular attention being paid to professional relationships and measurement of both the processes of work and outcomes of the patient. The length of the post-discharge follow-up period also should be extended.

\section{References}

1. Anthony MK, Hudson-Barr D (2004) A patient-centred model of care for hospital discharge. Clin Nurs Res 13:117.

2. Bowles KH, Naylor MD, Foust JB (2002) Patient characteristics at hospital discharge and a comparison of home care referral decisions. J Am Geriatr Soc 50: 336.

3. Chapin RK, Chandran D, Sergeant JF, Koenig TL (2014) Hospital to community transitions for adults: Discharge planners and community service providers' perspective. Soc Work Healthc 53: 311.

4. McMurray A, Johnson P, Wallis M, Patterson E, Griffths S (2007) General surgical patients' perspectives of the adequacy and appropriateness of discharge planning to facilitate health decision-making at home. J Clin Nurs 16:1602.

5. Bull MJ, Jervis LL (1997) Strategies used by chronically ill older women and their caregiving daughters in managing post hospital care. J Adv Nurs 25: 541 .

6. Coleman EA (2003) Falling through the cracks: Challenges and opportunities for improving transitional care for persons with continuous complex care needs. J Am Geriatr Soc 51: 549.

7. Laugaland K, Aase K, Barach P (2012) Interventions to improve patient safety in transitional care - A review of the evidence. Work 41: 2915.

8. MacAdam M (2008) Frameworks of integrated care for the elderly: A systematic review (Report).

9. Geary CR, Schumacher KL (2012) Care transitions: Integrating transition theory and complexity science concepts. ANS Adv Nurs Sci 35: 236.

10. Rooney M, Arbaje AI (2012) Changing the culture of practice to support care transitions - Why now? Generations 36: 63.

11. Boling PA (2009) Care transitions and home health care. Clin Geriatr Med 25: 135.

12. Forster AJ, Clark HD, Menard A, Dupuis N, Chernish R, et al. (2004) Adverse events among medical patients after discharge from hospital. CMAJ 170: 345-349.

13. Forster AJ, Murff HJ, Peterson JF, Gandhi TK, Bates DW (2003) The incidence and severity of adverse events affecting patients after discharge from the hospital. Ann Intern Med 138: 161.

14. Tsilimingras D, Bates DW (2008) Performance improvement. Addressing post-discharge adverse events: a neglected area. Jt Comm J Qual Patient Saf 34: 85 . 
15. Darwin B, Parrish M (2008) Navigating care transitions in California: Two models for change (Report).

16. Moore C, Wisnivesky J, Williams S, McGinn T (2003) Medical errors related to discontinuity of care from an inpatient to an outpatient setting. J Gen Intern Med 18: 646

17. Soares MM, Jacobs K, Laugaland K, Aase K, Barach P (2012) Interventions to improve patient safety in transitional care - A review of the evidence. Work 41: 2915.

18. Bauer M, Fitzgerald L, Haesler E, Manfrin M (2009) Hospital discharge planning for frail older people and their family. Are we delivering best practice? A review of the evidence. J Clin Nurs 18: 2539.

19. Block L, Morgan-Gouveia M, Levine RB, Cayea D (2014) We could have done a better job: A qualitative study of medical student reflections on safe discharge. J Am Geriatr Soc 62: 1147.

20. Hesselink G, Flink M, Olsson M, Barach P, Dudzik-Urbaniak E, et al. (2012) Are patients discharged with care? A qualitative study of perceptions and experiences of patients, family members and care providers. BMJ Qual Saf 21: i39-49.

21. Coleman EA, Boult C (2003) The American Geriatrics Society Health Care Systems Committee: Improving the quality of transitional care for persons with complex care needs. J Am Geriatr Soc 51: 556.

22. Enderlin CA, McLeskey N, Rooker JL, Steinhauser C, D’Avoilio D, et al. (2013) Review of current conceptual models and frameworks to guide transitions of care in older adults. Geriatr Nurs 34: 47-52.

23. Jack BW, Chetty VK, Anthony D (2009) A reengineered hospital discharge program to decrease re-hospitalization: A randomized clinical trial. Ann Intern Med 150: 178.

24. Naylor MD, Brooten D, Campbell R, Jacobsen BS, Mezey MD, et al. (1999). Comprehensive discharge planning and home follow-up of hospitalized elders: A randomized clinical trial. JAMA 281: 613-620.

25. Naylor MD, Brooten DA, Campbell RL, Maislin G, McCauley KM, et al. (2004) Transitional care of older adults hospitalized with heart failure: A randomized clinical trial. J Am Geriatr Soc 52: 675 .

26. Ouslander JG, Bonner A, Herndon L, Shutes J (2014) The interventions to reduce acute care transfers (INTERACT) quality improvement program: An overview for medical directors and primary care clinicians in long term care. J Am Med Dir Assoc 15:162.

27. Rennke S, Nguyen OK, Shoeb MH, Magan Y, Wachter RM, et al. (2013) Hospital-initiated transitional care interventions as a patient safety strategy: A systematic review. Ann Intern Med 158: 433.

28. Society of Hospital (2014) Society of Hosptial Medicine. Project BOOST: Better Outcome by Optimizing Safe Transitions.

29. Walraven CV, Dhalla IA, Bel C, Etchells E, Stiell IG, et al. (2010) Derivation and validation of an index to predict early death or unplanned readmission after discharge from hospital to the community. CMAJ 182: 551.

30. Friedman LM, Furberg CD, Demets DL (2010) Fundamentals of Clinical Trials (4th ed.) Springer, New York.

31. Rockwood K (2011) Videx FI-CGA (Version 1.2), Halifax, Canada.

32. Hawthorne G (2006) Measuring social isolation in older adults: Development and validation of the friendship scale. Soc Indicat Res 77: 521-548.

33. Coleman EA, Smith JD, Frank JC, Eilerst TB, Thiare JN, et al. (2002) Development and testing of a measure designed to assess the quality of care transitions. Int J Integr Care 2: 1.

34. Montori VM, Guyatt GH (2001) Intention-to-treat principle. CMAJ 165: 1339.
35. Mitnitski A, Mogilner AJ, Rockwood K (2001) Accumulation of deficits as a proxy measure of aging. ScientificWorldJournal 1: 323.

36. Dohan D, Schrag D (2005) Using navigators to improve care of underserved patients. Cancer 104: 848

37. Evans SJ, Sayers M, Mitnitski A, Rockwood K (2014) The risk of adverse outcomes in hospitalized older patients in relation to a frailty index based on a comprehensive geriatric assessment. Age Ageing 43: 127.

38. Rockwood K, Hogan D, Macknight C (2000) Conceptualisation and measurement of frailty in elderly people. Drugs Aging 17: 295.

39. Clegg A, Young J, Lliffe S, Rikkert MO, Rockwood K (2013) Frailty in elderly people. Lancet 381: 752.

40. Ornstein K, Smith KL, Foer DH, Lopez-Cantor M, Soriano T (2011) To the hospital and back home again: a nurse practitioner-based transitional care program for hospitalized homebound people. J Am Geriatr Soc 59: 544

41. Hansen LO, Young RS, Hinami K (2011) Interventions to reduce 30 day rehospitalization: A systematic review. Ann Intern Med 155: 520.

42. Michael YL, Berkman LF, Colditz GA, Kawachi I (2001) Living arrangements, social integration and change in functional health status. Am J Epidemiol 153: 123.

43. Russell D, Taylor J (2009) Living alone and depressive symptoms: The influence of gender, physical disability and social support among hispanic and non-hispanic older adults. J Gerontol B Psychol Sci Soc Sci 64B: 95.

44. Counsell SR, Callahan M, Clark DO, Tu W, Buttar AB, et al. (2007) Geriatric care management for low income seniors: A randomized control trial. JAMA 298: 2623.

45. Dy SM, Apostol C, Martinez KA, Aslakson RA (2013) Continuity, coordination and transitions of care for patients with serious and advanced illness: A systematic review of interventions. J Palliat Med 16 436.

46. Naylor MD, Aiken LH, Kurtzman ET, Olds DM, Hirschman KB (2011) The importance of transitional care in achieving health reform. Health Affairs 30: 746.

47. Parry C, Coleman EA, Smith JD, Frank J, Kramer AM (2003) The care transitions intervention: A patient-centered approach to ensuring effective transfers between sites of geriatric care. Home Health Care Serv Q 22: 1.

48. Reinhard SC, Lind KD (2011) Public policy: Implication for pathways through transitions: The rise of the transitional care concept. Gerontol Geriatr 31: 209.

49. Burke RF, Kripalani S, Vasilevskis EE (2013) Moving beyond readmission penalties: Creating an ideal process to improve transitional care. J Hosp Med 8: 102.

50. Nosbusch JM, Weiss ME, Bobay KL (2011) An integrated review of the literature on challenges confronting the acute care staff nurse in discharge planning. J Clin Nurs 20: 754.

51. Wagstaff N, Butler J, Kalanovic S, Shepley M (2010) High impact actions: discharge planning. Nurs Manag (Harrow) 17: 12

52. Doctoroff L, McNally D, Vanka A, Nall R, Mukamal KJ (2014) Inpatientoutpatient transitions for patients with resident primary care physicians: Access and readmission. Am J Med 127: 886 e15-20.

53. Misky GJ, Wald HL, Coleman EA (2010) Post-hospitalization transitions: Examining the effects of timing of primary care provider follow-up. J Hosp Med 5: 392. 